\title{
Controllable Fabrication of Composite Core-Shell Capsules at a Macroscale as Organoid Biocarriers
}

Fan He, ${ }^{\dagger, \&}$ Tingting Tao, ${ }^{\dagger,+, \&}$ Haitao Liu, ${ }^{\dagger,+}$ Yaqing Wang, ${ }^{\dagger,+}, \xi$ Kangli Cui ${ }^{\dagger,+}$ Yaqiong Guo, ${ }^{\dagger, t}$ and Jianhua Qin ${ }^{*},+,, ", \perp$

${ }^{\dagger}$ CAS Key Laboratory of Separation Science for Analytical Chemistry, Dalian Institute of Chemical Physics, Chinese Academy of Sciences, Dalian, 116023, P. R. China

University of Chinese Academy of Sciences, Beijing, 100049, P. R. China

${ }^{\S}$ School of Chemistry, Dalian University of Technology, Dalian 116024, P. R. China

"Institute for Stem Cell and Regeneration, Chinese Academy of Sciences, Beijing, 100101, P. R. China

${ }^{\perp}$ CAS Center for Excellence in Brain Science and Intelligence Technology, Chinese Academy of Sciences, Shanghai, 200031, P. R. China

*Corresponding Author. E-mail: jhqin@dicp.ac.cn (J.Q.)

${ }^{\&}$ These authors contributed equally to this work. 


\section{Supplementary Tables}

Table S1. Components of culture mediums for human islet organoids

\begin{tabular}{|c|c|c|}
\hline Culture medium & Basal medium & Components \\
\hline \multirow[t]{5}{*}{ DEM } & DMEM/F12 (Invitrogen) & $1 \% \mathrm{KSR}$ (Invitrogen) \\
\hline & & 1\% B27 supplement (Invitrogen) \\
\hline & & 50 ng/mL activin-A (PeproTech) \\
\hline & & 1\% GlutaMAX (Invitrogen) \\
\hline & & $1 \%$ penicillin-streptomycin (Invitrogen) \\
\hline \multirow[t]{7}{*}{ PEM } & DMEM (Invitrogen) & $0.5 \%$ B27 supplement (Invitrogen) \\
\hline & & $2 \mu \mathrm{M}$ dorsomorphin (Selleck) \\
\hline & & $2 \mu \mathrm{M}$ retinoic acid (RA; Sigma) \\
\hline & & $10 \mu \mathrm{M}$ SB431542 (Selleck) \\
\hline & & $5 \mathrm{ng} / \mathrm{mL}$ basic fibroblast growth factor, (bFGF; \\
\hline & & R\&D Systems) \\
\hline & & 250 nM SANT-1 (Selleck) \\
\hline \multirow[t]{5}{*}{ EPM } & DMEM (Invitrogen) & $0.5 \%$ B27 supplement (Invitrogen) \\
\hline & & $2 \mu \mathrm{M}$ dorsomorphin (Selleck) \\
\hline & & $10 \mu \mathrm{M}$ SB431542 (Selleck) \\
\hline & & $50 \mu \mathrm{g} / \mathrm{mL}$ ascorbic acid (Sigma) \\
\hline & & $10 \mu \mathrm{M}$ DAPT (Abcam Biochemicals) \\
\hline \multirow[t]{7}{*}{$\mathrm{ECM}$} & CMRL 1066 (Invitrogen) & $25 \mathrm{mM}$ glucose (Sigma) \\
\hline & & $0.5 \%$ B27 supplement (Invitrogen) \\
\hline & & 10 mM nicotinamide (Sigma) \\
\hline & & $10 \mu \mathrm{M}$ SB431542 (Selleck) \\
\hline & & $50 \mu \mathrm{g} / \mathrm{mL}$ ascorbic acid (Sigma) \\
\hline & & $2 \mu \mathrm{M}$ dorsomorphin (Selleck) \\
\hline & & $0.5 \%$ penicillin-streptomycin (Invitrogen) \\
\hline
\end{tabular}


Table S2. RT-PCR primer sequences

\begin{tabular}{ccc}
\hline Primer & Forward sequence $\left(5^{\prime} \rightarrow 3^{\prime}\right)$ & Reverse sequence $\left(5^{\prime} \rightarrow 3^{\prime}\right)$ \\
\hline INS & TGTACCAGCATCTGCTCCCTCTA & TGCTGGTTCAAGGGCTTTATTCCA \\
GCG & AGGCAGACCCACTCAGTGA & AACAATGGCGACCTCTTCTG \\
SST & CCCCAGACTCCGTCAGTTTC & TCCGTCTGGTTGGGTTCAG \\
PPY & ACCTGCGTGGCTCTGTTACT & TACCTAGGCCTGGTCAGCAT \\
PDX1 & CGTCCGCTTGTTCTCCTC & CCTTTCCCATGGATGAAGTC \\
NKX6.1 & ATTCGTTGGGGATGACAGAG & TGGGATCCAGAGGCTTATTG \\
NKX2.2 & TGGCCATGTAAACGTTCTGA & GGAAGAAAGCAGGGGAAAAC \\
\hline
\end{tabular}

Table S3. Primary antibody information

\begin{tabular}{cccc}
\hline Target & Dilution & Host & Company \\
\hline INS & $1: 1000$ & guinea pig & Abcam, ab7842 \\
SST & $1: 100$ & rat & Abcam, ab30788 \\
PPY & $1: 100$ & Goat & Abcam, ab77192 \\
GCG & $1: 100$ & rabbit & ThermoFisher, PA513442 \\
\hline
\end{tabular}




\section{Supplementary Figures}

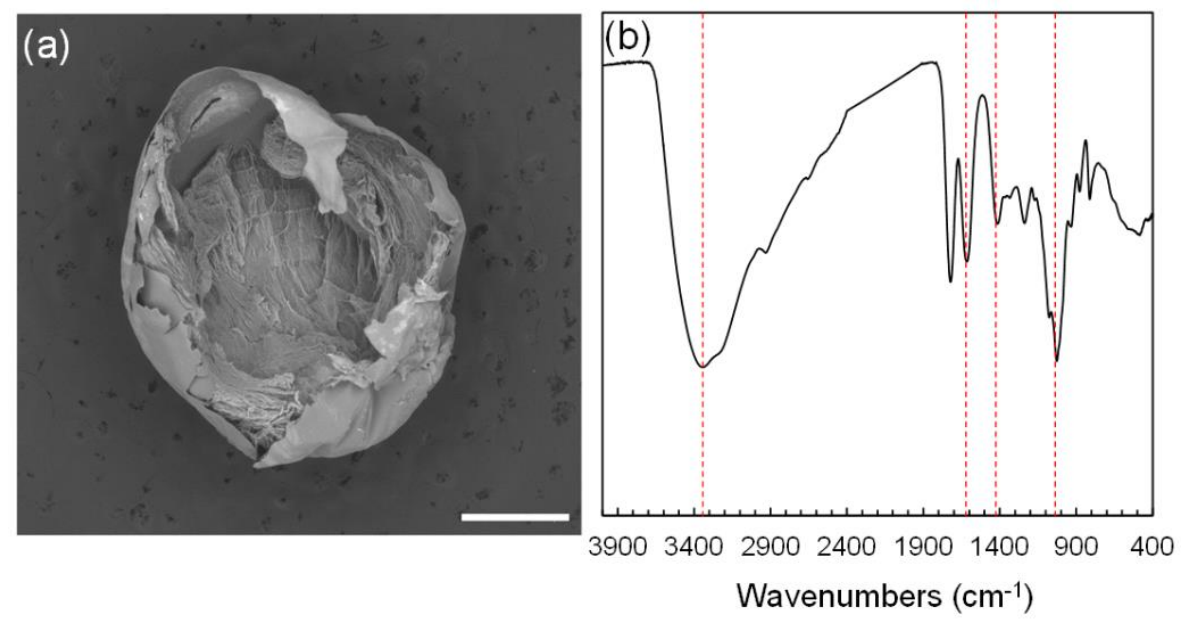

Figure S1. (a) SEM image showing the cross-section of Alg/PEI capsule. The scale bar is $1 \mathrm{~mm}$. (b) FT-IR spectrum of Alg/PEI capsule.
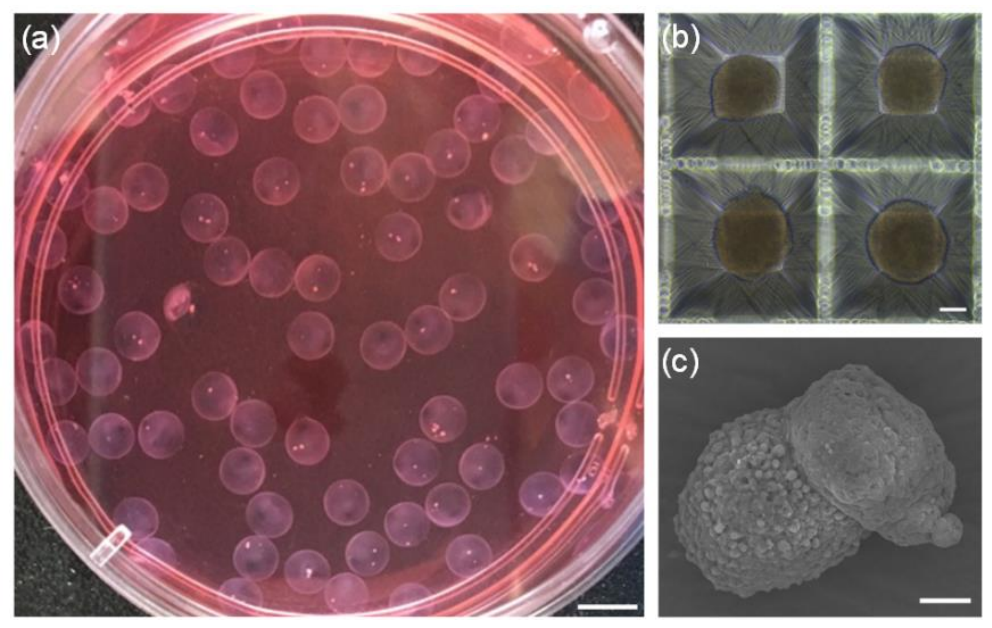

Figure S2. (a) Optical images of Alg/PEI composite macrocapsule carriers encapsulated with hiPSCs-derived spheroids inducted for 5 days (the end of DE stage) in PEM. (b,c) Optical (b) and SEM (c) images of hiPSCs-derived spheroids inducted for 5 days. The scale bars are $5 \mathrm{~mm}$ in (a), $100 \mu \mathrm{m}$ in (b) and $20 \mu \mathrm{m}$ in (c). 

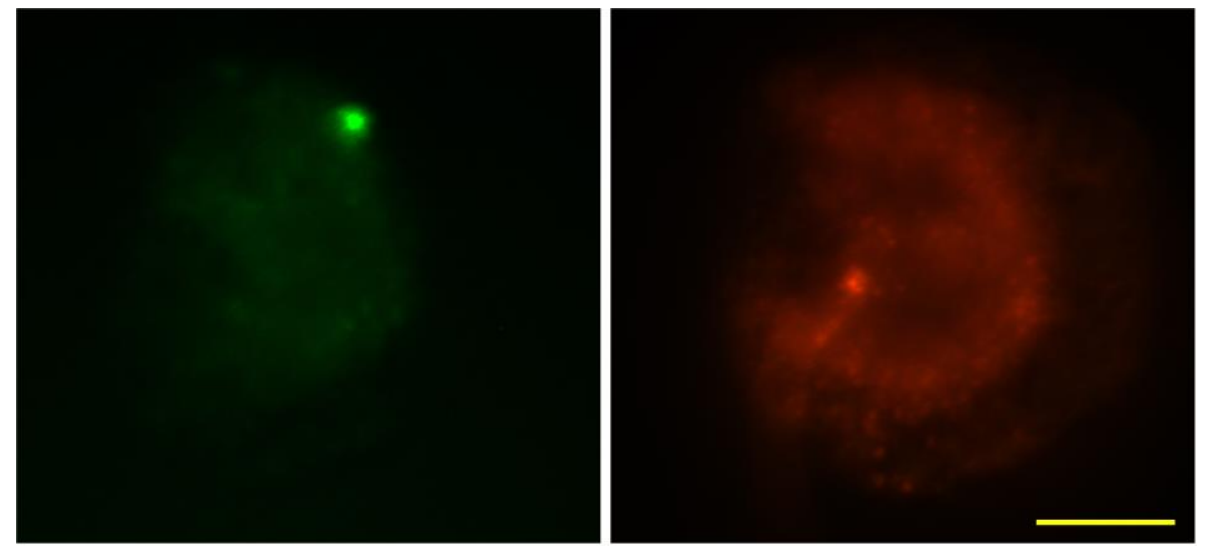

Figure S3. Fluorescent images showing the viability of an islet organoid after encapsulation in Ca-alginate macrobead hydrogel for 5 days. The scale bar is $100 \mu \mathrm{m}$. 